\title{
Alterstice
}

Revue internationale de la recherche interculturelle

International Journal of Intercultural Research

Revista International de la Investigacion Intercultural

\section{Rendre visible l'invisible : savoirs et prises de conscience de femmes immigrantes au sein d'organisations communautaires}

\section{Manon Chamberland et Yann Le Bossé}

Volume 4, numéro 1, 2014

URI : https://id.erudit.org/iderudit/1077480ar

DOI : https://doi.org/10.7202/1077480ar

Aller au sommaire du numéro

Éditeur(s)

Alterstice

ISSN

1923-919X (numérique)

Découvrir la revue

Citer cet article

Chamberland, M. \& Le Bossé, Y. (2014). Rendre visible l'invisible : savoirs et prises de conscience de femmes immigrantes au sein d'organisations communautaires. Alterstice, 4(1), 31-44. https://doi.org/10.7202/1077480ar
Résumé de l'article

Plusieurs études traitent des obstacles récurrents rencontrés par certains groupes de population, dont les femmes immigrantes, davantage confrontées à un marché du travail précaire et atypique. Il importe de réfléchir sur les pratiques destinées à soutenir l'intégration sociale et professionnelle de ces femmes, pour leur permettre à la fois d'exercer une influence sur leurs conditions de vie tout en reconnaissant leur expertise expérientielle. Cet article rend compte d'une recherche qualitative et exploratoire portant sur les pratiques au sein de certaines organisations communautaires propices à soutenir le développement du pouvoir d'agir des personnes et des collectivités. Les résultats mettent en évidence les savoirs que les femmes immigrantes qui fréquentent ces organisations développent et redécouvrent, ce qui contribue à l'exercice de changements à différents niveaux. 
ARTICLE HORS THÈME

\section{Rendre visible l'invisible : savoirs et prises de conscience de femmes immigrantes au sein d'organisations communautaires}

Manon Chamberland ${ }^{1}$ et Yann Le Bossé ${ }^{2}$

\section{Résumé}

Plusieurs études traitent des obstacles récurrents rencontrés par certains groupes de population, dont les femmes immigrantes, davantage confrontées à un marché du travail précaire et atypique. II importe de réfléchir sur les pratiques destinées à soutenir l'intégration sociale et professionnelle de ces femmes, pour leur permettre à la fois d'exercer une influence sur leurs conditions de vie tout en reconnaissant leur expertise expérientielle. Cet article rend compte d'une recherche qualitative et exploratoire portant sur les pratiques au sein de certaines organisations communautaires propices à soutenir le développement du pouvoir d'agir des personnes et des collectivités. Les résultats mettent en évidence les savoirs que les femmes immigrantes qui fréquentent ces organisations développent et redécouvrent, ce qui contribue à l'exercice de changements à différents niveaux.

\section{Rattachement des auteurs}

${ }^{1}$ Université du Québec en Outaouais, Campus de Saint-Jérôme, Saint-Jérôme (Québec), Canada

${ }^{2}$ Université Laval, Québec (Québec), Canada

\section{Correspondance}

manon.chamberland@uqo.ca

\section{Mots clés}

femmes immigrantes; développement du pouvoir d'agir des personnes et des collectivités; pratiques d'intervention sociale; groupe

\section{Pour citer cet article}

Chamberland, M. et Le Bossé, Y. (2014). Rendre visible l'invisible: savoirs et prises de conscience de femmes immigrantes au sein d'organisations communautaires. Alterstice, 4(1), 31-44. 


\section{Introduction}

La mondialisation et le développement exponentiel des connaissances et des technologies conduisent à diverses mutations sur le marché du travail (Bernier, 2011). De nombreuses études mettent en évidence les obstacles récurrents rencontrés par une partie de la population (jeunes, femmes, nouveaux immigrants, personnes moins scolarisées, etc.), qui peine à s'insérer socioprofessionnellement de manière satisfaisante (Beaudoin, 2012; Chicha, 2009; Malenfant, Lévesque, Jetté et White, 2004). Cela soulève des questions quant à ce que vivent ces personnes ainsi qu'aux modalités d'accompagnement mises en œuvre pour soutenir leurs démarches.

La période de transition qui accompagne l'immigration, les nombreuses démarches à effectuer pour faire reconnaître leurs compétences professionnelles ainsi que les différents rapports sociaux dans lesquels elles sont impliquées contribuent à ce que plusieurs femmes immigrantes soient confrontées, à des degrés divers, à une déqualification professionnelle (Chicha, 2009). Le taux de chômage des femmes immigrantes est deux fois plus élevé que celui des femmes natives des pays où elles s'établissent (OCDE, 2011). Leur revenu s'avère aussi moins élevé. Par exemple, au Canada, les femmes immigrantes gagnent en moyenne $9000 \$$ de moins que les hommes immigrants, qui ont eux-mêmes un revenu plus modeste que la population native (Conseil du statut de la femme, 2005; Mongeau, Pinsonneault et Rose, 2007). Bien que les recherches menées à propos des premières années d'installation des femmes immigrantes soulignent la diversité de leurs parcours, il semble qu'elles se retrouvent souvent dans une spirale d'obstacles alimentée par la déqualification et les emplois précaires, les horaires intermittents et les exigences particulières de la conciliation travail-famille (Chandler et Jones, 2003; Man, 2004). L'ensemble de ces facteurs est susceptible d'alimenter leur isolement social et d'avoir des conséquences sur leur santé physique et mentale (Bibeau, Chan-Yip, Lock, Rousseau et Sterlin, 1992; Conseil du statut de la femme, 2005; McMichael et Menderson, 2004), ce qui soulève de nombreux enjeux éthiques. En effet, devant le développement accéléré des connaissances, comment se fait-il qu'il soit acceptable qu'une bonne partie de la population soit toujours confrontée à la précarité? Comme le soulignent Bibeau et Fortin (2008) : " Nos sociétés respectent, c'est vrai, de plus en plus les différences culturelles mais cette ouverture au pluralisme ne les a pas protégées vis-à-vis d'autres inégalités, sociales et économiques surtout, qui se sont amplifiées au cours des dernières années " (p. 133). Les femmes immigrantes sont particulièrement touchées, notamment en raison de discriminations croisées (Chicha, 2009; Lefèvre, Boismenu et Dufour, 2011; Pierre, 2005).

Or les femmes immigrantes fréquentent peu les organisations de soutien (Benadj, 2005; Berger, 2004). Lorsqu'elles le font, le soutien est davantage centré sur des dimensions personnelles (comme l'estime de soi) plutôt que sur les défis quotidiens auxquels elles doivent faire face (Battaglini, 2008; Berger, 2004; Côté, Berteau, Durand, Thibaudeau et Tapia, 2002). Pourtant, les études portant sur les trajectoires des femmes immigrantes font ressortir diverses stratégies efficientes face à l'adversité, les compétences de ces femmes et le sentiment de fierté qu'elles en retirent (Breton, 1999; Chandler et Jones, 2003; Charrad, 2010; Cloutier, 2011; Lisboa, 2002; Zentgraf, 2002). Ce dernier constat conduit à s'interroger sur la manière de tenir compte de l'expérience spécifique des femmes immigrantes. Cela rejoint également ce que certains auteurs mentionnent concernant les trajectoires conduisant vers l'exclusion et la pauvreté, soit la nécessité de tenir compte non seulement des conditions qui y concourrent mais aussi de la manière dont ces situations sont vécues " de l'intérieur " (Groulx, 2011; Malenfant, Lévesque, Jetté et White, 2004). Cela ne signifie nullement qu'il faille négliger de s'interroger sur les conditions sociales qui y contribuent, mais plutôt qu'il faille bien se garder de conclusions hâtives et stéréotypées de ce que constituent les inégalités sociales pour les personnes qui les vivent et de ce que sont les stratégies pour les contrecarrer. Depuis plusieurs décennies, un intérêt soutenu pour l'empowerment dans de nombreuses disciplines permet d'envisager ce processus comme une alternative pertinente, en raison de ses retombées à la fois personnelles et sociales.

\section{L'empowerment : un processus et des pratiques}

Depuis le début du $20^{\mathrm{e}}$ siècle, plusieurs mouvements sociaux (mouvements pour des résidences sociales, pour les droits civiques aux États-Unis, mouvements féministes, des gais et des lesbiennes, mouvements des personnes vivant avec un handicap et mouvement de l'anti-psychiatrie, etc.) ont permis de mieux comprendre la manière dont des personnes durablement touchées par des conditions inégalitaires et la stigmatisation parvenaient à s'en affranchir (Cox et Parsons, 2000; Rappaport, 1987; Simon, 1996). L'empowerment, ce processus désignant le 
passage d'une forme d'impuissance à son affranchissement, a suscité un engouement particulier, et ce, dans différentes disciplines. Plus particulièrement en travail social (Breton, 1999, 2002 et 2004; Gutiérrez, 1990 et 1994 ; Gutiérrez et Lewis, 1999; Lee, 2001; Ninacs, 2008) et en psychologie communautaire (Rappaport, 1987; Le Bossé, 2004; Le Bossé et Dufort, 2001), les recherches ont permis de mieux circonscrire ce processus, ainsi que de conceptualiser certains principes valables pour l'élaboration d'un cadre de pratiques destiné aux intervenants désireux de soutenir dans cette perspective les populations. Bien que la définition de l'empowerment en tant que processus varie selon les contextes (Rappaport, 1987), Guttiérrez (1990 et 1994) distingue un certain nombre de manifestations communes qui se développent dans l'action : l'augmentation du sentiment d'efficacité personnelle et de la confiance, la redécouverte ou le développement de nouvelles habiletés ainsi que le développement d'une conscience critique. Pour Ninacs (2008), l'empowerment comprend quatre dimensions qui se renforcent mutuellement : la participation, les composantes pratiques (c'est-à-dire les compétences et les habiletés pratiques requises par la participation et l'action), l'estime de soi et la conscience critique. Cette dernière dimension est conditionnelle à la prise de conscience d'un problème vécu qui confronte la personne à une forme d'impuissance (Hur, 2005), ce que Ninacs (2008) désigne comme la prise de conscience individuelle. La participation à une initiative avec d'autres personnes conduirait à une conscience collective (la personne n'étant plus la seule à avoir le problème), à une conscience sociale (les problèmes sont influencés par la manière dont la société est organisée) et à une conscience politique (la solution aux problèmes structurels nécessite un changement politique). L'engouement pour l'empowerment a conduit à définir, pour les praticiens désireux de soutenir ce processus chez les populations qu'ils accompagnent (qu'il s'agisse de personnes ou de collectivités), un cadre formé d'un ensemble de composantes interreliées.

La première de ces composantes consiste à s'intéresser prioritairement aux personnes aux prises avec les conséquences des inégalités sociales (Lee, 2001; Simon, 1996). En effet, pour qu'il y ait empowerment, il doit y avoir au préalable prise de conscience d'un problème constituant une forme d'impuissance. Celle-ci prendra différentes formes selon les contextes, ce qui constitue la deuxième composante (Le Bossé, 2004; McWhirter, 1998). Une fois réalisée la prise de conscience d'un problème vécu individuellement, s'instaurent un dialogue et un rapport fondé sur la collaboration (Freire, 1976), ce qui constitue la troisième composante. Cela replace la personne ou la collectivité dans une position d'expertise, ce qui constitue la quatrième composante (Breton, 2004; Gutiérrez, 1990; McWhirter, 1998). Au fil de ce processus se développe une conscience critique, la cinquième composante, ce qui permet de voir en quoi les conditions structurelles interagissent avec les situations vécues personnellement, et ce qui révèle des pistes d'action là où il y avait initialement une source d'impuissance (Lee, 2001; McWhirter, 1998; Ninacs, 2008). Ces diverses composantes ont été considérées par plusieurs auteurs, notamment en travail social (Lee, 2001) et en counseling (McWhirter, 1998), comme aptes à fournir une approche guidant les intervenants. Du côté francophone, les travaux de Le Bossé (2004, 2010 et 2012) ont permis non seulement de proposer une traduction et des précisions conceptuelles importantes, mais aussi de développer un cadre d'analyse des pratiques destiné aux différents acteurs intéressés par l'empowerment. Nous retenons ici pour nos travaux l'expression "développement du pouvoir d'agir» des personnes et des collectivités » pour désigner le processus de l'empowerment. Selon les dimensions qui caractérisent ce phénomène, le développement du pouvoir d'agir des personnes et des collectivités soulève la question des savoirs que détiennent les personnes. Quels sont ces savoirs et comment s'arriment-ils à ceux des intervenants? En effet, si les intervenants visent à soutenir le développement du pouvoir d'agir des personnes et des collectivités, une appréhension plus circonscrite des savoirs engendrés par un tel processus semble pertinente.

Qu'en est-il du développement du pouvoir d'agir des femmes lors de la période de transition que constitue l'immigration? Les recherches sur le sujet sont plutôt rares (Chamberland, 2007; Zentgraf, 2002). Cependant, certaines recherches ayant porté sur l'expérience de la migration, par exemple de la campagne à la ville (Erman, Kalaycioglu et Rittersberger-Tillic, 2002; Lisboa, 2002), et sur des initiatives menées par des femmes immigrantes pour exercer une influence sur des situations limitant leurs droits (Breton, 1999; Chandler et Jones, 2003) ont permis d'attirer l'attention sur l'importance de ce que les femmes immigrantes font. Alors que l'expérience du travail salarié à l'extérieur n'était pas nouvelle pour plusieurs femmes (Erman et collab., 2002; Zentgraf, 2002), la fréquentation d'espaces de médiation, comme par exemple un groupe ou des associations permettant aux femmes de se regrouper, d'échanger des informations et des savoir-faire, d'élargir leur réseau de connaissances et de développer une perception différente de leur situation, serait significative. Pour certaines, le fait d'occuper de 
nouveaux rôles dans ces groupes les inciterait à renégocier les rôles au sein de leur famille (Lisboa, 2002) ou faire valoir leurs droits dans des sphères où elles n'osaient pas le faire auparavant (Chandler et Jones, 2003). Ce processus serait aussi fortement rattaché à l'importance qu'elles accordent à leur famille (Chamberland, 2007; Erman et collab., 2002; Zentgraf, 2002). À ce titre, s'intéresser plus précisément aux pratiques au sein des organisations communautaires constitue une piste pertinente pour révéler les savoirs développés par les femmes immigrantes et la manière dont ceux-ci s'arriment à ceux des intervenants lorsqu'il est question de développement du pouvoir d'agir. En effet, constituant historiquement des lieux propices à l'action collective, les organisations communautaires ont régulièrement été associées au développement du pouvoir d'agir, bien que les transformations survenues ces dernières décennies (professionnalisation, partenariats avec l'État) aient soulevé plusieurs interrogations à cet égard (Duval, Fontaine, Fournier, Garon et René, 2005; Le Goff, McAll et Montgomery, 2005; René, 2009).

\section{Méthode}

Dans le but de mieux cerner cette question, nous avons mené une recherche exploratoire basée sur l'étude de cas multiples pour documenter l'expérience des intervenantes et celle des femmes immigrantes au sein de diverses organisations communautaires, deux situées à Montréal, deux à Bruxelles et une à Grenoble.

\section{Sélection des cas}

La première étape a consisté à établir, à partir des répertoires régionaux des services sociaux de Montréal, une liste de 67 organisations communautaires fréquentées par des femmes immigrantes. Dans un deuxième temps, cette liste a été réduite à 25 organisations communautaires, à la suite de l'analyse des informations disponibles à l'aide d'une grille de six critères ${ }^{1}$ construite à partir de l'idéal-type des organismes communautaires de René (2005), ainsi que des modèles de Rifkin (2003) et de McWhirter (1998) ${ }^{2}$. Dans un troisième temps, ces 25 organisations communautaires ont été hiérarchisées selon leur potentiel illustratif du développement du pouvoir d'agir, à partir de l'analyse approfondie des informations disponibles sur Internet. À la suite d'un appel téléphonique, deux des cinq premières organisations communautaires de la liste ont accepté de participer à la recherche. Une première organisation, fondée il y a plus de 15 ans par des femmes d'origines diverses souhaitant avoir un lieu de rencontre et d'échanges, rassemble aujourd'hui plus de 250 membres de plus de 67 nationalités différentes. Les femmes y viennent pour faire partie de comités et prendre part à des activités ponctuelles (org. A). La deuxième organisation communautaire est issue d'un projet-pilote mis sur pied il y a quelques années pour offrir à des femmes immigrantes l'opportunité de se former pour devenir accompagnantes des familles nouvellement arrivées. Pour ce faire, pendant dix mois, elles bénéficient d'une formation tout en étant rémunérées (org. B).

Afin de diversifier les contextes, trois autres organisations communautaires, recommandées par des informateursclés et satisfaisant aux critères de la grille d'analyse, ont été ajoutées au corpus initial. Deux organisations sont situées à Bruxelles et ont été fondées dans les années 1970. Elles concentrent leurs activités dans le secteur de l'alphabétisation et de l'éducation permanente (éducation populaire). L'une d'entre elles est exclusivement fréquentée par des femmes d'origines étrangères (org. C), l'autre est mixte et s'adresse tant aux Belges de naissance que d'origine étrangère (org. D). Enfin, la cinquième organisation, située à Grenoble, a été créée en 2009. Elle s'adresse aux mères qui éduquent seules leurs enfants. Ces dernières y viennent pour échanger et prendre part à des activités avec leurs enfants dans une atmosphère détendue (org. E). Au moment de l'entretien, toutes les femmes qui fréquentaient cet endroit étaient immigrantes.

\footnotetext{
${ }^{1}$ Ces critères sont la possibilité de s'impliquer dans des activités propices à utiliser ses forces et ses compétences; la reconnaissance de sa dignité et de ses droits; la possibilité de pouvoir exercer un changement dans sa situation personnelle à partir de sa propre définition du changement; la possibilité de pouvoir participer, d'une manière ou d'une autre, aux instances décisionnelles; la possibilité de s'impliquer et de connaître d'autres personnes; la possibilité de pouvoir participer à des activités qui rassemblent aussi bien des personnes immigrantes que d'autres membres de la population (communauté géographique ou d'intérêt).

2 Deux autres modélisations ont été ciblées au départ, celles de Le Bossé (2004) et de Lee (2001). Cependant, celle de McWhirter s'est avérée la plus pertinente et opérationnelle pour sélectionner les organisations communautaires et analyser les informations disponibles.
} 


\section{Collecte de données}

Au total, 13 intervenantes (dont 5 coordonnatrices et 1 directrice, que nous nommons toutes "intervenantes " dans la suite du texte) ont été rencontrées dans le cadre d'entretiens semi-dirigés individuels de 50 à 90 minutes. Toutes ont lu et signé le formulaire de consentement portant sur les objectifs de la recherche, sur la participation attendue et sur les précautions concernant la confidentialité. Un entretien de groupe a permis de rencontrer entre 3 et 8 femmes dans chaque organisation communautaire. Tous les entretiens ont été enregistrés et retranscrits et les données ont été anonymisées.

\section{Analyse des données}

Plusieurs étapes d'analyse du contenu manifeste (lectures successives, codage ligne par ligne, etc.) ont permis de répondre aux questions initiales. Chaque entrevue avec les intervenantes et les femmes immigrantes a été résumée en une à deux pages. L'analyse des entretiens en groupe (ligne par ligne puis par résumé) a permis de valider les thèmes émergents des pratiques des intervenantes et de faire ressortir les savoirs que les femmes retirent de leur fréquentation de ces organisations communautaires. Enfin, un tableau reprenant chaque catégorie a permis d'effectuer une comparaison entre les sites.

\section{Résultats}

Quels savoirs les femmes immigrantes ont développés ou redécouverts dans ces organisations communautaires, et en quoi ceux-ci contribuent à l'exercice d'un changement face aux obstacles qu'elles rencontrent? Ces savoirs se situent à différents niveaux : elles ont pu effectuer une relecture de leur propre histoire et mieux se connaître, approfondir leurs connaissances sur leur milieu de vie, leur quartier et le pays dans lequel elles se sont établies et enfin établir des liens entre leurs conditions de vie et la manière dont la société est organisée, en ce qui concerne non seulement les obstacles rencontrés mais aussi les ressources disponibles susceptibles de soutenir leurs démarches. Ces ressources incluent des forces insoupçonnées qu'elles avaient en elles. Mais c'est surtout au moyen des relations développées avec d'autres femmes qu'elles ont renouvelé leur regard sur le monde et sur les possibilités de s'y inscrire.

\section{Différents savoirs transmis et redécouverts}

Parmi les éléments qui ressortent des propos des femmes immigrantes et des intervenantes figurent résolument le développement ou la redécouverte de savoirs qui contribuent à ce qu'elles se perçoivent différemment, tout comme les possibilités d'agir face à des situations au premier abord insolubles. Par exemple, alors que le fait de ne pas maîtriser la langue d'usage dans le pays d'accueil peut être d'abord perçu comme une limite, les femmes découvrent au contraire qu'elles possèdent un atout pour accompagner les familles.

La langue. Avant on ne l'utilisait pas et on la gardait pour nous mais on l'utilise maintenant pour venir en aide aux personnes. (Mirela, Montréal, org. B)

Dans les organisations $C$ et $D$, alors que ne pas savoir lire et écrire peut être stigmatisant, les femmes redécouvrent les compétences qu'elles détiennent et parlent surtout de ce qu'elles savent et veulent accomplir. Comment s'effectuent ces recadrages?

\section{Relire sa propre histoire et mieux se connaître}

En fréquentant les organisations communautaires, les femmes ont accès à une foule d'informations, mais c'est en pouvant les rattacher concrètement à leur expérience que ces informations deviennent significatives. En participant à un groupe, les femmes sont invitées à partager leurs expériences tout en expérimentant de nouveaux rôles. Par exemple, dans l'organisation A, les femmes ont la possibilité d'être co-animatrices, de prendre part à des groupes d'entraide, de participer à des comités pour préparer des initiatives de mobilisation. Dans l'organisation B, les femmes immigrantes expérimentent le fait d'être accompagnantes et sont placées en position d'aidantes et de personnes-ressources. Lors de leur formation, elles ont donc l'occasion de revisiter leur propre histoire d'immigration, notamment en découvrant les ressources de leur quartier et en prenant connaissance des rouages 
de la société d'accueil pour les nouveaux arrivants. Dans l'organisation C, elles participent à des groupes pour se former et elles ont aussi l'occasion d'organiser et de prendre en charge différents projets qui les mettront en contact avec différents acteurs de la collectivité. C'est plus spécifiquement dans l'échange de leurs connaissances avec les autres que les femmes redécouvrent leurs savoirs.

\section{Transférer ses connaissances et les partager}

Ce partage de connaissances permet notamment aux femmes immigrantes de prendre conscience de ce qu'elles ont développé. Par exemple, dans l'organisation B, la formation des femmes-accompagnantes débute par une visite des différentes ressources de quartier à Montréal. Puisque deux groupes de femmes-accompagnantes par année se chevauchent, les femmes du premier groupe peuvent côtoyer les femmes du second groupe lors des accompagnements, pour en réaliser progressivement certains de manière autonome, au fur et à mesure de l'avancement de leur formation. Ces femmes peuvent ainsi transférer ce qu'elles ont appris aux autres. Dans l'ensemble des organisations communautaires participantes s'effectue donc un partage des savoirs, où coexistent différentes manières de voir le monde. Les femmes rencontrées font le constat d'une diversité de perspectives.

[...) je suis ici depuis janvier seulement, mais ce sont des ouvertures, ce sont des portes ouvertes mais dans la compréhension de l'autre, pas simplement moi qui analyse avec mon système, mais c'est l'autre qui s'explique. (Maryvonne, Montréal, org. A)

En échangeant les unes avec les autres, par exemple dans le cadre de groupes de soutien, dans des ateliers d'écriture ou encore dans le cadre de projets artistiques où elles ciblent un sujet et mettent en commun leurs savoirs expérientiels ${ }^{3}$ pour écrire une histoire, elles sont amenées à revoir les moments décisifs de leur parcours ou encore à mesurer les changements qu'elles remarquent à ce jour dans leur quotidien. Mais leurs apprentissages ne se limitent pas à une meilleure connaissance d'elles-mêmes.

\section{Approfondir la connaissance de son environnement}

À travers les initiatives auxquelles elles participent, les femmes immigrantes apprennent aussi à connaître leur milieu de vie ainsi que la manière dont la société est organisée.

Maintenant si les gens parlent d'économie ou de l'école, la crise, tout ça, maintenant je sais qu'est-ce qu'ils parlent, quoi. Avant je ne sais pas, je suis comme quelqu'un qui est sourd [...]. (Roberta, Bruxelles, org. D)

Par exemple, dans l'organisation $D$, des ateliers dans le cadre de l'université populaire permettent de réunir des professeurs d'université experts de certaines questions (économie, éducation, emploi, etc.), le groupe de femmes, les formatrices en alphabétisation et des membres de la collectivité. Le travail de synthèse qu'elles ont à réaliser en petits groupes permet, d'une part, de dégager ce qu'elles retiennent de la présentation effectuée et, d'autre part, de rattacher ces informations à leur vécu quotidien. Ainsi, une retombée significative de ce type d'initiative est qu'elles se sentent davantage en mesure de revendiquer leurs droits et de conduire leur vie selon ce qu'elles souhaitent pour elles et leur famille.

[...] je suis contente parce que aussi au niveau de l'école, [...] ils disent aussi : " Si un enfant ne maîtrise pas bien, c'est vite orienté ". Là aussi je connais maintenant, si je ne suis pas d'accord et je veux que ma fille continue d'étudier, j'ai le droit d'insister. (Sophia, Bruxelles, org. D)

En fait, pour certaines organisations, développer de telles connaissances était à l'origine de leur fondation. Cela est particulièrement le cas de l'organisation $\mathrm{C}$, où les femmes cherchaient à obtenir davantage d'information sur le fonctionnement de leur société d'accueil, pour l'aide aux devoirs, etc. Or, ce qui se dégage des entrevues réalisées,

\footnotetext{
${ }^{3}$ Ces savoirs issus de l'expérience vécue forment l'« expertise expérientielle » (De Goer, Ferrand et Hainzelin, 2008). À l'instar de Borkman (1976), dont les travaux sont consacrés aux groupes d'entraide, cette expression désigne l'expertise issue de l'expérience d'un phénomène et non provenant d'informations reçues par d'autres moyens tels que des lectures, des recherches, une formation, etc.
} 
c'est que ce n'est pas tant dans une critique de la manière dont la société est organisée que circulent les informations mais bien dans la découverte des ressources pour atteindre leurs objectifs.

\section{Un regard différent sur ses habiletés et sur les sources de soutien disponibles}

Les retombées identifiées par les femmes immigrantes rencontrées dans cette recherche concernent simultanément les plans personnel, interpersonnel et politique. C'est-à-dire qu'en se percevant différemment ainsi qu'en prenant conscience des alternatives pour faire face aux obstacles rencontrés, les femmes développent non seulement un sentiment d'appartenance au groupe mais aussi une vision du monde différente.

Ça m’a ouvert les yeux. [...] je pensais ne jamais prendre la parole et maintenant avec le temps, je peux prendre la parole et parler facilement, mais je me suis sentie, je sentais que j'étais bien entourée. (Marie, Bruxelles, org. D)

Elles retrouvent aussi, au sein des organisations et dans les différentes initiatives auxquelles elles prennent part, une reconnaissance qui les amène à changer le regard qu'elles portent sur elles-mêmes et sur leurs possibilités de s'inscrire dans la collectivité.

\section{Une reconnaissance par les autres}

Les femmes immigrantes vivent une reconnaissance des savoirs qu'elles détiennent de la part des différents interlocuteurs qu'elles côtoient. Par exemple, les collaborations établies par l'organisation B avec d'autres organisations dans le quartier ont permis aux personnes qui y travaillent et à la population de connaître les ressources que les femmes détiennent et mettent à la disposition de la collectivité.

Nous avions dernièrement un forum là à [cette école], bien toutes les enseignantes qui étaient présentes là, elles venaient voir, pour [nous] saluer parce qu'on leur apporte vraiment un appui très très fort. Oui parce qu'on parle plusieurs langues, français, on parle anglais, arabe, créole, tamoul, vietnamien, espagnol, donc y a plusieurs cultures et c'est très riche en informations. (Yvonne, Montréal, org. B)

Par la rencontre avec d'autres acteurs de la collectivité, elles perçoivent qu'elles peuvent exercer concrètement une influence, comme en témoigne l'exemple suivant. Les autorités responsables du transport en commun sont venues rencontrer les femmes afin de connaître quelles contraintes elles constataient dans leur quartier. Leurs propos ont ainsi pu donner lieu à des modifications significatives afin que le quartier soit mieux desservi par les transports en commun.

Moi, je te dis, de sentir que ce qu'on dit est considéré et qu'on peut faire une différence, ça ouvre vers de nouvelles possibilités. (Khalida, Montréal, org. B)

Par le plus grand sentiment d'influence qu'elles ressentent, à travers les habiletés qu'elles redécouvrent et développent dans le cadre des organisations communautaires participantes, elles en viennent à percevoir une plus grande liberté par leurs actions.

Oui, surtout parce que quand tu ne sais pas lire ni écrire un moment tu sais lire et écrire sans demander [à] quelqu'un ça donne de la joie [...]. C'est comme si avant on était attachée et puis tout à coup, on est détachée. Ça donne la liberté. (Marine, Bruxelles, org. D)

Ces retombées semblent indissociables du processus qui se développe dans le groupe et au cours duquel les femmes se lient à d'autres femmes et définissent leur réussite non seulement en des termes personnels, mais également en la reliant à la réussite des autres.

Y avait un lien entre les personnes. Chacun encourage l'autre. Chacun encourage l'autre. Voilà. Tu vas y arriver, tu vas le faire, vas-y, même s'il dit non je vais pas y arriver ou bien je sais pas, on a un encouragement dans tout le groupe pour que tout le monde avance. (Liliane, Bruxelles, org. C) 
Cette solidarité établie entre les femmes devient une ressource supplémentaire pour faire face aux défis qu'elles rencontrent. Grâce à leur participation à ces organisations communautaires, elles retrouvent également des ressources personnelles, dont une motivation décuplée et un courage redécouvert.

\section{Une motivation décuplée}

Les femmes mentionnent plusieurs retombées personnelles. Elles font en particulier état d'une motivation qui fait en sorte qu'elles ne veulent plus s'arrêter :

C'est comme un oiseau qui apprend à marcher et là debout pour marcher, et puis il a commencé à faire comme ça (elle fait le mouvement de battre des ailes) et à voler un petit peu, il a vu tout le coin. Maintenant, il a envie de voler sans arrêt. (Rhonda, Bruxelles, org. D)

Elles se sentent déterminées, ce qui est aussi bien visible dans leur entourage.

[...] j'avais de la gêne vraiment ancrée en moi et puis petit à petit je me suis dit : « Je peux travailler, je suis capable autant qu'une autre, ça a eu des conséquences et ça a vraiment fait un déclic dans ma tête [...] beaucoup l'ont vu dans mon entourage, mes enfants, mon mari, mes sœurs, tout le monde a remarqué ce changement. [...] Maintenant je sais ce que je veux et voilà c'est ça et on ne me fera pas changer d'idée. (Liliane, Bruxelles, org. C)

\section{Un courage redécouvert}

Au contact des autres et dans le cadre des initiatives auxquelles elles prennent part, elles redécouvrent un courage qu'elles avaient parfois oublié.

Moi, c'est comme quand moi je suis venue ici, c'était comme une petite graine, un très très petite graine et puis le [nom de l'association] m'a arrosée et je suis devenue une fleur parce que enfin ça a réveillé quelque chose en moi parce que pendant 5-6 ans que j'étais à la maison, je ne faisais plus rien. C'est comme si ça m'avait donné le courage de pouvoir aller plus loin. (Liliane, Bruxelles, org. C)

Ces retombées font en sorte qu'elles sont en mesure de se projeter dans l'avenir.

\section{Formuler des projets}

Une des retombées commune à l'ensemble des organisations est que les femmes sont davantage en mesure de formuler des projets. En témoignent les propos de cette femme, au terme de sa formation en alphabétisation :

Oui, je veux continuer ma formation, mes études jusqu'à l'université! Ça c'est mon rêve maintenant. (Rhonda, Bruxelles, org. D.)

Non seulement, pour certaines, elles recommencent à rêver, mais elles savent aussi sur quelles ressources s'appuyer pour réaliser leurs projets.

C'est vraiment une grande porte qui est ouverte pour nous et puis bon, moi, ça m'aide. C'est vrai que les gens disent que je suis forte dans la tête mais seule, c'est à plusieurs qu'on devient plus forte encore. (Marie-Jeanne, Grenoble, org. E)

Le sentiment d'appartenance développé par leurs implications en groupe et la solidarité dont elles font preuve avec d'autres femmes se traduisent aussi par le fait qu'elles se disent davantage concernées, curieuses et ouvertes sur le monde.

[...] notre camarade nous avait raconté ce qui s'était passé dans son pays, on se sent un peu concernée tout de même, du coup cet événement on le voit autrement. Parce que quand on entend parler du génocide, du coup on pense à notre amie, elle avait perdu sa famille, elle a laissé sa famille derrière elle. On se sent concernée quelque part. (Rita, Bruxelles, Org. C) 
Ainsi, elles rattachent leur réussite non seulement à l'atteinte d'objectifs personnels mais bien à aux réussites des autres femmes. Dans l'organisation C, une femme a mentionné qu'il s'agit en fait d'une réussite politique si les femmes dégagent de ces expériences vécues en groupe le sentiment qu'elles peuvent réussir à se tailler une place, à s'insérer socioprofessionnellement selon leurs désirs et, surtout, sans être arrêtées par des obstacles insurmontables qui confinent à l'impuissance et à la résignation. Les initiatives auxquelles les femmes prennent part ont des retombées bien au-delà de leur vie personnelle. Les intervenantes soulignent à quel point les femmes immigrantes qu'elles rencontrent savent créer des liens.

Elles savent déjà mettre en lien des gens c'est important aussi. (Béatrice, intervenante, Bruxelles, org. C)

À la base de leur fréquentation des organisations communautaires, les femmes souhaitaient déjà d'une manière ou d'une autre s'impliquer. Cependant, décrivant les changements réalisés entre le moment où elles ont souhaité le faire (ce qui a pu motiver le début de leur implication) et le moment de l'entrevue, elles s'expriment en termes de contrastes visibles.

Moi je dirais que j'étais dans le brouillard et que maintenant, je vois bien. (Marie, Bruxelles, org. C)

Moi j'étais dans le coma. (rires) Franchement, ces dix ans, j'ai rien fait, c'était le coma total. Y a quelque chose qui s'est passé et je suis en vie. Tout simplement. (Sonia, Bruxelles, org. C)

Ces contrastes rendent compte du fait que leur vision du monde dans lequel elles s'insèrent et, surtout, leur vision de ce qu'elles souhaitent y faire ont changé.

Ça m’a ouvert les yeux. (Liliane, Bruxelles, org. C)

\section{Discussion}

Quels savoirs ont développé les femmes immigrantes rencontrées lors de leur passage dans ces organisations communautaires? En quoi ceux-ci permettent-ils de dépasser les obstacles qui se dressent devant elles? Les propos des femmes incitent à penser que le processus de travail en groupe a une grande importance, ce qui rejoint ce que d'autres auteurs ont avancé (Breton, 1999, 2004; Carr, 2003 et 2004). Celui-ci devient un espace médiateur : les femmes peuvent y expérimenter de nouveaux rôles, être dans une position où elles partagent des informations et des savoirs avec les autres. Elles développent des relations basées sur la réciprocité, si bien que leur réussite est rattachée à celle des autres. Cependant, il ne s'agit pas tant de considérer que seul le fait de réunir des femmes immigrantes suffirait à développer un pouvoir d'agir. Cette étude rend compte du fait qu'en rejoignant un groupe, les femmes immigrantes ont l'occasion de relire leur propre histoire et de redécouvrir les savoirs qu'elles détiennent déjà. Elles mettent en commun non seulement leurs histoires et les obstacles qu'elles rencontrent, mais aussi les compétences qu'elles détiennent dans la réalisation d'un projet. Bref, leurs propos laissent penser que plusieurs dynamiques d'aide mutuelle ${ }^{4}$ se sont développées dans le groupe (Steinberg, 2008). Dans le cadre d'un groupe avec des femmes d'origine chinoise qui ont migré de la campagne à la ville, Hung (2012) mentionne que le partage des histoires personnelles des femmes au début du groupe a fait en sorte de créer un sentiment d'universalité de leurs expériences, permettant de réduire la culpabilité ressentie face aux obstacles qu'elles rencontraient. Raconter leurs histoires leur a donc permis de rétablir une continuité entre " l'avant » la migration et "l'après ". De plus, elles ont eu la possibilité dans le groupe de co-construire un discours alternatif qui remplace les stéréotypes qui leur sont généralement attribués. Leurs forces, leurs compétences et leurs préoccupations ont ainsi été mises de l'avant, ce qui a contribué à une redéfinition de leur identité (Hung, 2012). Or, au sein des organisations que nous avons étudiées, il semble que ce ne soit pas tant le partage d'expériences que celui de la réalisation d'un projet qui se soit révélé important pour les femmes.

\footnotetext{
${ }^{4}$ Les neufs dynamiques d'aide mutuelle sont, selon Steinberg (2008), le partage d'information, la confrontation des idées, la discussion de sujets tabous, tous et toutes dans le même bateau, le soutien émotionnel, les demandes mutuelles, l'aide à la résolution de problèmes individuels, l'expérimentation de façons d'être et de faire et enfin la force du nombre.
} 
C'est aussi aux opportunités qu'elles découvrent dans leur environnement que l'on peut rattacher le processus de développement du pouvoir d'agir. Cet aspect est moins souvent mis en évidence. En effet, Hung (2012) mentionne plutôt, comme point de départ à la redéfinition de l'identité de ces femmes, une perspective critique de la manière dont la société est organisée. Ce qui ressort des résultats de notre recherche serait davantage que ce qui est important pour ces femmes est de prendre part à des initiatives dont les retombées leur donnent le sentiment de contribuer à la collectivité, et que l'atteinte de leurs objectifs personnels soit rattachée à celle des autres femmes. Elles tissent des liens et redécouvrent leur environnement, qui n'est alors plus seulement limitatif mais aussi pourvoyeur de ressources auxquelles elles peuvent recourir pour réaliser leurs rêves. Les propos des femmes rencontrées laissent penser qu'elles envisagent leur participation à la collectivité comme offrant davantage de sens, notamment en raison des projets qu'elles formulent et qui leur permettent de s'inscrire dans celle-ci.

Selon Breton (2012), une telle prise de conscience peut avoir plusieurs retombées pour la poursuite d'une plus grande justice sociale, car les personnes transfèrent les habiletés qu'elles ont développées au sein du groupe à la collectivité, en dégageant des pistes constructives pour agir. C'est peut-être même en ce sens que l'on peut parler de développement du pouvoir d'agir des personnes et des collectivités. Les retombées personnelles sont transférables sur les plans interpersonnel et politique. Comme le souligne Breton (2012), cela nécessite parfois quelques petits pas de plus pour les travailleurs afin de mettre en place les conditions de réalisation à l'extérieur du groupe. Cela implique donc de dépasser les sphères personnelles et interpersonnelles en tant que visée, afin de faciliter le processus.

Un autre aspect significatif qui émerge des résultats de notre recherche est la dimension publique des actions posées par les femmes. En effet, côtoyer d'autres acteurs qui ne font pas partie de l'organisation communautaire semble particulièrement significatif en ce qui concerne la reconnaissance de leurs compétences. Il semblerait que cela soit même constitutif du passage entre les espaces médiateurs que constituent le groupe et l'organisation communautaire vers la collectivité. D'autres recherches portant sur les trajectoires des personnes qui ont pris part à des groupes et qui ont fréquenté des organisations communautaires permettraient de formuler des hypothèses plus précises quant à l'effet significatif d'accomplir une action vue et reconnue par autrui. II demeure que les actions auxquelles prennent part les femmes immigrantes semblent significatives à cet égard. Par exemple, dans l'organisation $A$, les membres d'un groupe de soutien en violence conjugale se sont mobilisées pour écrire un texte racontant leur histoire et leur processus d'affranchissement, qui a été publié dans le journal local. Dans l'organisation B, les actions des femmes accompagnantes sont de plus en plus reconnues par les institutions avec lesquelles les familles arrivantes transigent. Dans l'organisation C, un service de garde a été mis en place par et pour les femmes désirant poursuivre une formation mais qui n'avaient pas accès aux crèches, réservées aux personnes qui travaillent. Cette initiative a fait l'objet d'un documentaire présenté, entre autres, au ministre. Dans l'organisation $\mathrm{D}$, plusieurs projets pédagogiques ont une dimension publique (théâtre, capsules radio, université populaire).

Bref, à différents égards, l'ensemble de ces initiatives contribue, petit à petit, à changer les représentations des uns et des autres. Y contribuent aussi les liens d'amitié créés au sein du groupe et qui perdurent même une fois que la formation est terminée. Cela rejoint ce que Duffy (2011) remarque à propos de mères célibataires qui ont participé à une recherche-action-participation. Même plusieurs mois après le projet, des liens d'amitié demeurent et s'approfondissent au fil du temps. Comme le souligne Freire (1998), un des premiers savoirs nécessaires à la conduite du changement est celui " d'être avec », qui permet la solidarité et de se voir comme capable d'exercer une influence.

Cette recherche comporte quelques limites. Parmi celles-ci figure le fait que nous avons rencontré exclusivement des femmes pour qui la fréquentation des organisations communautaires semble avoir été significative. Or cela peut ne pas être toujours le cas, ce qui soulève la question du moment où se produit cette fréquentation. $S^{\prime}$ intéresser aux trajectoires de femmes immigrantes permettrait d'avancer un peu plus quant à ces deux aspects.

Alterstice - Revue Internationale de la Recherche Interculturelle, vol. 4, $n^{\circ} 1$ 


\section{Conclusion}

Devant les obstacles récurrents rencontrés par les femmes immigrantes, notamment en ce qui concerne leur insertion socioprofessionnelle, il est nécessaire de s'interroger sur les pratiques d'accompagnement qui visent à soutenir leurs initiatives. Bien que la pertinence de l'approche centrée sur le développement du pouvoir d'agir soit régulièrement mentionnée, les connaissances à son sujet demeurent parcellaires. En particulier, bien qu'il soit établi que le processus de développement du pouvoir d'agir donne lieu à la redécouverte ou au développement de savoirs, leur description et les liens que l'on peut établir avec les différents changements qui lui sont attribuables sont toujours à documenter. À cet égard, s'intéresser plus particulièrement aux pratiques au sein d'organisations communautaires qui s'adressent aux femmes immigrantes s'est avéré pertinent. Cela a permis de révéler plusieurs savoirs développés par des femmes immigrantes au sein de ces organisations communautaires et de comprendre en quoi ceux-ci contribuent au changement face aux obstacles rencontrés par ces femmes. Les résultats montrent que ces organisations, leur fonctionnement et les pratiques qui y sont privilégiées permettent de rendre visible l'invisible, et ce, à différents niveaux.

D’abord, les femmes immigrantes y redécouvrent des forces insoupçonnées et des sources de soutien, par la rencontre avec d'autres femmes avec lesquelles elles partagent non seulement des similitudes, mais aussi des différences, différences qui se révèlent propices aux débats et aux apprentissages. Par leur participation concrète à des actions rattachées à leurs objectifs, ces femmes en viennent à considérer leurs conditions sociales non seulement comme constituant des obstacles, mais aussi comme contribuant à soutenir leurs initiatives. Dans ces organisations communautaires, les femmes en arrivent à percevoir que quelque chose est possible, qu'elles peuvent exercer un changement avec d'autres afin de s'affranchir pour avancer.

Ce processus d'affranchissement est rattaché non seulement au regard que les femmes immigrantes portent sur elles-mêmes et sur leur situation, mais aussi au regard des autres acteurs de la collectivité, ce qui est susceptible non seulement de contribuer à soutenir leurs démarches mais aussi de créer des liens propices au changement social et aux apprentissages réciproques. En ce sens, à un autre niveau, les savoirs issus de ces différents contextes de rencontre se développent non seulement chez les femmes immigrantes, mais aussi chez les intervenantes et chez d'autres acteurs dans la collectivité. Les femmes immigrantes comme les intervenantes contribuent en ce sens chaque jour au changement social, par le développement de connaissances partagées. Ces connaissances s'enracinent dans l'expérience quotidienne, invitant à revoir les modalités de financement de ces organisations et à tenir compte de ce qui est bien visible: certes, les conséquences de la prégnance des thèses associées au néolibéralisme peuvent entraîner un éloignement des individus les uns des autres, mais certaines pratiques au sein des organisations communautaires contribuent à soutenir concrètement les initiatives des femmes immigrantes, en soulignant les savoirs qu'elles détiennent déjà ainsi que les retombées des actions qu'elles entreprennent au quotidien.

\section{Références bibliographiques}

Battaglini, A. (2008). Exclusion et santé. Le rôle de la santé publique vis-à-vis des populations immigrantes. Dans É. Gagnon, Y. Pelchat et R. Édouard (dir.), Politiques d'intégration, rapports d'exclusion, action politique et justice sociale (p. 187-199). Québec : Presses de I’Université Laval.

Beaudoin, P. (2012). Portrait des femmes immigrées des minorités visibles recensées au Québec en 2006 et de leur participation au marché du travail. Québec : Ministère de l'Immigration et des Communautés culturelles.

Benhadj, L. (2005). Portrait des femmes immigrantes fréquentant certains organismes publics et communautaires de services de santé et de services sociaux de la Capitale-Nationale. Québec : Agence de développement de réseaux locaux de services de santé et de services sociaux de la Capitale-Nationale.

Berger, R. (2004). Immigrant women tell their stories. New York : Haworth Press.

Bernier, C. (2011). Formation et employabilité, regard critique sur l'évolution des politiques de formation de la main-d'œuvre au Québec. Québec : Presses de l'Université Laval. 
Bibeau, G. et Fortin, S. (2008). Inégalités et souffrance sociale : Une approche anthropologique. Dans K. Frolich, M. De Koninck, A. Demers et P. Bernard (dir.), Les inégalités sociales de santé au Québec (p. 111-139). Montréal : Presses de l’Université de Montréal.

Bibeau, G., Chan-Yip, A., Lock, M., Rousseau, C. et Sterlin, C. (1992). La santé mentale et ses visages, un Québec pluriethnique au quotidien. Montréal : Gaëtan Morin.

Borkman, T. (1976). Experiential knowledge : a new concept for the analysis of self-help groups. Social service review, 50(3), 445-456.

Breton, M. (1999). The relevance of structural approach to group work with immigrant and refugee women. Social work with groups, 22(2-3), 11-29.

Breton, M. (2002). Empowering practice in Canada and the United States : restoring policy issues at the center of social work. The Social Policy Journal, 1(1), 19-34.

Breton, M. (2004). An empowerment perspective. Dans C. Garvin, L. Guttiérrez et M. Galinsky (dir.), Handbook of Social Work with Groups (p. 58-75). New York: The Guilford Press.

Breton, M. (2012). Small steps toward social justice. Social work with groups, 35, 205-217.

Carr, E. (2003). Rethinking empowerment theory using a feminist lens: the importance of process. Affilia, 18(1), 8-20.

Carr, S. (2004). Accessing resources, transforming systems. Dans C. Garvin, L. Gutiérrez et M. Galinsky (dir.), Handbook of social work with groups (p. 360-383). New York : The Guilford Press.

Chamberland, M. (2007). Étude du développement du pouvoir d'agir à travers des parcours d'intégration de femmes nouvelles arrivantes à Montréal (mémoire de maîtrise). Université du Québec à Montréal, Canada.

Chandler, S. et Jones, J. (2003). "You Have to Do It for The People Coming": Union Organizing and the Transformation of immigrant woman workers. Affilia, 18(3), 254-271.

Charrad, M. (2010). Women's agency across cultures: conceptualizing strenghts and boundaries. Women's studies international forum, 33, 517-522.

Chicha, M.-T. (2009). Le mirage de l'égalité : les immigrées hautement qualifiées à Montréal (rapport de recherche). Montréal : Immigration et Métropoles.

Conseil du statut de la femme (2005). Des nouvelles d'elles, les femmes immigrées au Québec. Québec : Gouvernement du Québec.

Cloutier, G. (2011). La valorisation des savoirs de femmes immigrantes en milieu communautaire. Montréal : Richard Vézina.

Côté, B., Berteau, G., Durand, D., Thibaudeau, M. et Tapia, M. (2002). Empowerment et femmes immigrantes (rapport de recherche). Montréal : Direction de santé publique.

Cox, E. et Parsons, R. (2000). Empowerment-Oriented Practice : from practice value to practice model. Dans P. Allen-Meares et C. Garvin (dir.), The handbook of social work direct practice (p. 113-129). Thousand Oaks : Sage Publications.

De Goer, B., Ferrand, C. et Hainzelin, P. (2008). Croisement des savoirs : une nouvelle approche pour les formations sur la santé et la lutte contre les exclusions. Santé publique, 2(20), 163-175.

Duffy, L. (2011). "Step-by-Step We are Stronger": Women's Empowerment Through Photovoice. Journal of Community Health Nursing, 28(2), 105-116.

Duval, M., Fontaine, A., Fournier, D., Garon, S. et René, J.-F. (2005). Les organismes communautaires au Québec : Pratiques et enjeux. Montréal : Gaétan Morin. 
Erman, T., Kalaycioglu, S. et Rittersberger-Tillic, H. (2002). Money-earning activities and empowerment experiences of rural migrant women in the city : the case of Turkey. Women's studies international forum, 25(4), 395-410.

Freire, P. (1998). Pedagogy or freedom, ethics, democracy and civic courage. Lanham, Maryland : Rowman and Littlefield.

Groulx, L.-H. (2011). Les facteurs engendrant l'exclusion au Canada. Québec : Centre d'étude sur la pauvreté et l'exclusion (CEPE). http://www.cepe.gouv.qc.ca/publications/pdf/CEPE_lionel-henri_groulx.pdf

Guttiérrez, L. (1990). Working with women of color : an empowerment perspective. Social work, 35, $149-153$.

Gutiérrez, L. (1994). Beyond coping: an empowerment perspective on stressful life events. Journal of sociology and social welfare, 21(3), 201-219.

Guttiérrez, L. et Lewis, E. (1999). Empowering women of color. New York : Columbia University Press.

Hung, S. (2012). Empowerment group for women migrating from China to Hong Kong. Social work with groups, 35(1), 4-17.

Hur, M. (2005). Empowerment in terms of theoretical perspectives: exploring a typology of the process and components across disciplines. Journal of community psychology, 34(5), 523-540.

Le Bossé, Y. (2004). De "l'habilitation » au « pouvoir d'agir » : vers une appréhension plus circonscrite de la notion d'empowerment. Nouvelles pratiques sociales, 16(2), 30-51.

Le Bossé, Y. (2010). Champs d'application et finalités des pratiques en sciences de l'orientation: un point de vue psychosociologique. Québec : Éditions ARDIS.

Le Bossé, Y. (2012). Sortir de l'impuissance. Invitation à soutenir le développement du pouvoir d'agir des personnes et des collectivités. Québec : Éditions ARDIS.

Le Bossé, Y. et Dufort, F. (2001). Le cadre paradigmatique de la psychologie communautaire : vers une épistémologie du changement social. Dans F. Dufort, F. Guay, et J.-M. Bouchard (dir.), Agir au cœur des communautés : la psychologie communautaire et le changement social (p. 33-74). Sainte-Foy : Presses de I’Université Laval.

Lee, J. (2001). The empowerment approach to social work practice. New York : Columbia University Press.

Lefèvre, S., Boismenu, G. et Dufour, P. (2011). La pauvreté, quatre modèles sociaux en perspective. Montréal : Presses de l’Université de Montréal.

Le Goff, F., McAll, C., Montgomery, C. (2005). La transformation du communautaire. Montréal : Éditions SaintMartin.

Lisboa, T. (2002). Migrant women in south Brazil and their process of empowerment. Migration, 39-41, 113-135.

Malenfant, R., Lévesque, M., Jetté, M. et White, D. (2004). Étude de trajectoires liées à la pauvreté. Québec : Recherches sur les impacts psychologiques, organisationnels et sociaux du travail (RIPOST).

Man, G. (2004). Gender, work and migration : deskilling Chinese immigrant women in Canada. Women's studies international forum, 27, 135-148.

McMichael, C. et Manderson, L. (2004). Somali women and well-being: social networks and social capital among immigrant women in Australia. Human organization, 63(1), 88-99.

McWhirter, E. (1998). An empowerment model of counsellor education. Canadian journal of counseling/Revue canadienne de counseling, 32(1), 12-16.

Mongeau, J., Pinsonneault, G. et Rose, D. (2007). Portrait économique des femmes immigrées recensées au Québec. Québec : Ministère de l'immigration et des communautés culturelles.

Ninacs, W. (2008). Empowerment et intervention. Québec : Presses de l’Université Laval. 
Organisation de coopération et de développement écononiques (OCDE) (2011). Perspectives des migrations internationales 2011. Paris : OCDE.

Pierre, M. (2005). Les facteurs d'exclusion faisant obstacle à l'intégration socioéconomique de certains groupes de femmes immigrées au Québec. Nouvelles pratiques sociales, 17(2), 75-94.

Rappaport, J. (1987). Terms of empowerment / Examplars of prevention: Toward a theory for community psychology. American journal of community psychology, 15(2), 121-148.

René, J.-F. (2005). Les organismes communautaires. Dans M. Duval, A. Fontaine, D. Fournier, S. Garon et J.-F. René (dir.), Les organismes communautaires au Québec : pratiques et enjeux. Montréal : Gaétan Morin.

René, J.-F. (2009). L'individualisation de l'intervention: levier ou barrière à la prise en charge démocratique? Nouvelles pratiques sociales, 22(1), 111-124.

Rifkin, S. (2003). A framework linking community empowerment and health equity: it is a matter of CHOICE. Journal of health population nutrition, 21(3), 168-180.

Simon, B. (1996). The empowerment tradition in social work history. New York : Columbia University Press.

Steinberg, D. (2008). Le travail de groupe. Un modèle axé sur l'aide mutuelle. Québec: Presses de l'Université Laval.

Zentgraf, K. (2002). Immigration and women's empowerment, Salvadorans in Los Angeles. Gender and society, 16(5), 625-646. 